\title{
CANONICAL OBJECTS IN KIRILLOV THEORY ON NILPOTENT LIE GROUPS
}

\author{
RICHARD C. PENNEY
}

\begin{abstract}
It is shown that to each element $f$ in the dual space of the Lie algebra of a nilpotent Lie group there is a uniquely defined subgroup $K_{\infty}$ for which the representation corresponding to $f$ is inducible from a squareintegrable-modulo-its-kernel representation of $K_{\infty}$.
\end{abstract}

I. Introduction. Let $G$ be a connected, simply connected nilpotent Lie group with Lie algebra $\mathfrak{A}$. Let $U$ be an irreducible unitary representation of $G$. Under the Kirillov correspondence $U$ corresponds to a unique orbit $\theta$ of the co-adjoint representation $\mathrm{ad}^{*}$ of $G$ in the dual space $\mathfrak{U}^{*}$. The correspondence is obtained by selecting an $f$ in $\theta$ and a polarization for $f$ and then forming an appropriate induced representation (see [1]). The polarizations corresponding to a given $f$ are highly nonunique and noncanonical. In this paper we ask the following question: To what extent is it possible to describe $U$ by means of objects canonically defined by $\theta$ (or $f$ )? Our main result is the following theorem.

THEOREM. Associated with each $f \in \mathcal{O}$ there is a canonical subalgebra $\mathcal{K}_{\infty}$ with the following properties:

(a) If $K_{\infty}$ is the corresponding subgroup to $\mathcal{K}_{\infty}$ and $U^{\infty}$ is the representation of $K_{\infty}$ corresponding to $f \mid \mathcal{K}_{\infty}$ then $\operatorname{ind}\left(K_{\infty}, G, U^{\infty}\right)$ (the representation induced by $\left.U^{\infty}\right)$ is irreducible and equivalent to $U$.

(b) $K_{\infty}$ is invariant under any automorphism which fixes $f$.

(c) $U_{\infty}$ is square integrable modulo its kernel (see [4]).

This theorem seems useful from several points of view. $\mathcal{K}_{\infty}$ of course must contain polarizations for $f$. Thus there is a distinguished class of polarizations. Furthermore, let $\mathcal{K}_{\infty}$ be the radical of $f \mid \mathcal{K}_{\infty}$ i.e.

$$
\mathcal{H}_{\infty}=\left\{X \in \mathscr{K}_{\infty} \mid f\left(\left[X, \mathscr{K}_{\infty}\right]\right)=\{0\}\right\} .
$$

Then $f\left(\left[\mathcal{H}_{\infty} \mathcal{H}_{\infty}\right]\right)=0$. Let $H_{\infty}$ be the corresponding subgroup. Then exp if $\circ \log \mid H_{\infty}=\chi_{\infty}$ defines a character of $H_{\infty}$. Since $U^{\infty}$ is square integrable modulo its kernel, ind $\left(H_{\infty}, K_{\infty}, \chi_{\infty}\right)$ is primary and quasi-equivalent to $U^{\infty}$ (see [4]). Hence $\operatorname{ind}\left(H_{\infty}, G, \chi_{\infty}\right)$ is primary and quasi-equivalent to $U$. The subgroups $H_{\infty}$ can be used to describe in a canonical fashion the primary

Received by the editors June 10, 1976.

AMS (MOS) subject classifications (1970). Primary $22 \mathrm{E} 45$. 
projections onto the primary subspaces of nilmanifolds in much the same way that polarizations are used in the character formulas of [2]. We shall go into this in a later paper.

II. Proofs. In this section we shall define and study $K_{\infty} . K_{\infty}$ is defined by means of $\mathcal{H}_{\infty}$. We shall at first assume only that $G$ is a connected, simply connected, solvable Lie group. This will complicate some of our proofs but we feel that it sheds more light on the subject.

Let $f \in \mathfrak{U}^{*}$ and let $O(f)$ be its orbit under ad*. Let

$$
\delta(f)=\left\{\lambda \in \mathfrak{U}^{*} \mid \mathcal{O}(f)+t \lambda=\mathcal{O}(f) \text { for all } t \in \mathbf{R}\right\} .
$$

$\delta(f)$ is a subspace of $\mathfrak{U}^{*}$ which is invariant under $\operatorname{ad}^{*}$. Let $\mathscr{H}(f)$ $=\cap \operatorname{ker} \lambda(\lambda \in \delta(f))$.

LEMMA 1. $\mathcal{H}(f)$ is an ideal in $\mathfrak{A}$.

Proof. $\mathcal{H}(f)$ is invariant under $\operatorname{ad}(\exp t X)$ for all $X \in \mathfrak{A}$. Differentiating we see $[X, \mathcal{H}(f)] \subset \mathcal{H}(f)$. Q.E.D.

Now we define a sequence of subalgebras of $\mathfrak{A}$ as follows:

$$
\mathcal{H}_{1}(f)=\mathscr{H}(f), \quad \mathcal{H}_{k}(f)=\mathcal{H}_{(}\left(f \mid \mathcal{H}_{k-1}(f)\right) .
$$

Let $\mathcal{H}_{\infty}(f)=\cap \mathcal{H}_{k}(f)(k \in \mathbf{N})$.

LEMMA 2. Let $\mathfrak{X}_{1}$ and $\mathfrak{U}_{2}$ be Lie algebras. Let $A: \mathfrak{U}_{1} \rightarrow \mathfrak{U}_{2}$ be an automorphism and let $f_{2} \in \mathfrak{A}_{2}^{*}$ and $f_{1} \in \mathfrak{A}_{1}^{*}$ be such that $f_{2} \circ A=f_{1}$. Then $\mathcal{H}_{\infty}\left(f_{2}\right)$ $=A\left(\mathcal{H}_{\infty}\left(f_{1}\right)\right)$.

Proof. Clearly $A^{*}\left(\delta\left(f_{2}\right)\right)=\delta\left(f_{1}\right)$. Hence $A\left(\mathcal{H}_{1}\left(f_{1}\right)\right)=\mathcal{K}_{1}\left(f_{2}\right)$. The result follows by induction since $\mathcal{H}_{\infty}(f)=\mathscr{K}_{\infty}\left(f \mid \mathcal{H}_{1}\right)$. Q.E.D.

We shall require a criterion for deciding when $\mathcal{H}_{\infty}=\mathfrak{A}$. First we need some notation. If $f \in \mathfrak{U}^{*}$ and $\mathfrak{N}$ is subspace of $\mathfrak{U}$, let

$$
\mathfrak{N}^{f}=\{X \in \mathfrak{U} \mid f([X, M])=0 \text { for all } M \in \mathfrak{N}\} .
$$

Let $\Re=\mathfrak{U}^{f}$. Let $R=\{x \in G \mid \operatorname{ad} x f=f\}$.

THEOREM $1 . \mathcal{K}_{\infty} \neq \mathfrak{A}$ iff there is a proper ideal $g$ of $\mathfrak{A}$ containing $\Re$.

Proof. $\mathcal{H}_{\infty} \neq \mathfrak{U}$ iff $\mathcal{H}_{1} \neq \mathfrak{A}$, so it suffices to consider $\mathcal{K}_{1}$. We claim that $\mathcal{K}_{1}$ contains $\Re$. Let

$$
K=\left\{x \in G\left|\operatorname{ad}^{*} x(f)\right| \mathcal{K}_{1}=f \mid \mathcal{K}_{1}\right\} .
$$

Since $\mathcal{H}_{1}^{\perp}$ (the annihilator of $\mathcal{H}_{1}$ ) is $\delta$, we have $f+\mathcal{H}_{1}^{\perp} \subset \mathcal{O}(f)$. It follows that $\operatorname{ad}^{*} K(f)=f+\mathcal{K}_{1}^{\perp}$. Hence $\operatorname{dim} R \backslash K=\operatorname{dim} \mathcal{K}_{1}^{\perp}$. The Lie algebra $\mathscr{K}$ of $K$ is $\mathcal{H}_{1}^{f}$. Then $\operatorname{dim} \mathscr{K}-\operatorname{dim} \Re=\operatorname{dim} R \backslash K=\operatorname{dim} \mathcal{H}^{\perp}$. The kernel of the map $X \rightarrow f([X, \cdot])$ of $\mathscr{K}$ into $\mathfrak{U}^{*}$ is $\Re$ and hence the image of $\mathscr{K}$ has $\operatorname{dim} \mathscr{K}-\operatorname{dim} \mathscr{R}$ $=\operatorname{dim} \mathcal{H}_{1}^{\perp}$. The image is contained in $\mathcal{H}_{1}^{\perp}$ so 


$$
\mathscr{K}_{1}^{\perp}=\{f([X, \cdot]) \mid X \in \mathscr{K}\} .
$$

Hence $\mathcal{K}_{1}=\mathcal{K}^{f}$ and thus $\Re \subset \mathcal{K}_{1}$, proving that $\Re$ is contained in an ideal.

Conversely, let $R_{0}$ be the subgroup corresponding to $\Re$. If $g$ is an ideal containing $\Re$, we claim that $g^{\perp} \subset \delta(f)$. Let $\mathcal{L}=g^{f}$. Let $L$ be the corresponding analytic subgroup of $G$. The mapping $\phi: x \rightarrow \operatorname{ad}^{*} x(f)$ of $L$ into $\theta(f)$ maps into the affine subspace $f+g^{\perp}$ and is constant on cosets of $R_{0}$ in $L$. (Note that $R_{0} \subset L$.) It gives rise to a $C^{\infty}$ map $\tilde{\phi}$ of $R_{0} \backslash L$ into $f+g^{\perp}$. The tangent space at $R_{0} e$ in $R_{0} \backslash L$ is canonically isomorphic with $\Re \backslash \mathcal{L}$ while the tangent space at $f$ in $f+g^{\perp}$ is $g^{\perp}$. The differential of $\tilde{\phi}$ at $R_{0} e$ is given by $X+\Re \rightarrow f([X, \cdot])$. Now the bilinear form $B_{f}=f([\cdot, \cdot])$ is nondegenerate on $\mathfrak{R} \backslash \mathfrak{U}$ and hence $\operatorname{dim} \mathfrak{R} \backslash \mathfrak{A}-\operatorname{dim} \Re \backslash g=\operatorname{dim} \mathscr{R} \backslash \mathcal{L}$ since $\mathcal{L}=g^{f}$. It follows that $\operatorname{dim} \Re \backslash £=\operatorname{dim} g^{\perp}$ so $d \tilde{\phi}$ is surjective at $R_{0} e$. Hence $d \tilde{\phi}$ is nonsingular at $R_{0} e$ and $\tilde{\phi}$ is an open map in a neighborhood of $R_{0} e$. In particular $\operatorname{ad}^{*} L(f)$ contains a neighborhood of $f$ in $f+g^{\perp}$. Similar comments hold for $f$ replaced by $\operatorname{ad}^{*} g(f), g \in L$. It follows that ad* $L(f)$ is open in $f+g^{\perp}$. The same is true for any $f^{\prime} \in f+g^{\perp}$ so the orbits of $L$ in $f+g^{\perp}$ are all open. Since different orbits are disjoint and $f+g^{\perp}$ is a union of orbits, this contradicts the connectedness of $f+g^{\perp}$ unless there is only one orbit. Hence $\operatorname{ad}^{*} L(f)$ $=f+g^{\perp}$, showing that $f+g^{\perp} \subset \mathcal{O}(f)$. Similarly $f^{\prime}+g^{\perp} \subset \mathcal{O}(f)$ for any $f^{\prime} \in O(f)$. It follows that $g^{\perp} \subset \delta(f)$. Q.E.D.

Recall that a subalgebra $\mathcal{K}$ of $\mathfrak{A}$ is said to be subordinate to $f$ if $f([\mathcal{H}, \mathcal{H}])=0$.

COROllaRY. If $\mathfrak{A}$ is nilpotent, $\mathcal{H}_{\infty}$ is subordinate to $f$.

Proof. Let $f^{\prime}=f \mid \mathcal{H}_{\infty}$. By definition of $\mathcal{H}_{\infty}, \mathcal{H}_{\infty}\left(f^{\prime}\right)=\mathcal{H}_{\infty}$. Hence there is no proper ideal $\mathscr{g}$ containing $\mathcal{R}\left(f^{\prime}\right)$. Since $\mathcal{H}_{\infty}$ is nilpotent, this implies that $\Re\left(f^{\prime}\right)=\mathcal{H}_{\infty}$. Obviously $\Re\left(f^{\prime}\right)$ is subordinate. Q.E.D.

Definition. Let $\mathcal{K}_{\infty}=\left(\mathcal{H}_{\infty}\right)^{f} \cap$ normalizer $\left(\mathcal{K}_{\infty}\right)$. Let $K_{\infty}$ be the corresponding connected analytic subgroup.

Proposition. $\mathcal{H}_{\infty}=\left(\mathcal{K}_{\infty}\right)^{f}$ and $\mathrm{ad}^{*} K_{\infty}(f)=f+\mathcal{H}_{\infty}^{\perp}$.

Proof. Let $\mathscr{K}_{1}=\mathcal{H}_{1}^{f}$ and let $K_{1}$ be the corresponding subgroup. For $k \in K_{1}, \operatorname{ad}^{*}(k) f\left|\mathcal{H}_{1}=f\right| \mathcal{H}_{1}$. Hence ad $k\left(\mathcal{H}_{\infty}\right)=\mathcal{H}_{\infty}$ by Lemma 2. (Recall $\mathcal{H}_{\infty}=\mathcal{K}_{\infty}\left(f \mid \mathcal{H}_{1}\right)$.) It follows that $\mathcal{K}_{1} \subset \mathcal{K}_{\infty}$. Let $\mathcal{K}_{n}(f)=\mathcal{K}_{1}\left(f \mid \mathcal{H}_{n-1}\right)$. (Note that $\mathscr{K}_{n}(f) \subset \mathcal{K}_{n-1}(f)$.) Then $\Sigma_{i} \mathcal{K}_{i} \subset \mathscr{K}_{\infty}$ and $\mathscr{K}_{\infty}^{f} \subset \cap_{i} \mathcal{K}_{i}^{f}$. As was shown in the proof of the above theorem, $\mathcal{K}_{1}^{f}=\mathcal{K}_{1}$. Hence $\mathcal{K}_{i}^{f} \cap \mathcal{K}_{i-1}=\mathcal{K}_{i}$. It follows that $\cap_{i} \mathcal{K}_{i}=\cap_{i} \mathcal{K}_{i}=\mathcal{K}_{\infty}$. Hence $\mathcal{K}_{\infty}^{f} \subset \mathcal{K}_{\infty}$. Obviously $\mathcal{K}_{\infty}$ $\subset \mathcal{K}_{\infty}^{f}$ so we have shown $\mathcal{K}_{\infty}^{f}=\mathcal{K}_{\infty}$. That $\operatorname{ad}^{*} K_{\infty}(f)=f+\mathcal{K}_{\infty}^{\perp}$ is the same as the argument that $\operatorname{ad}^{*} K_{1} f=f+\mathcal{H}_{1}^{\perp}$ done in the above theorem. Q.E.D.

COROLlaRY. In the notation of the above proof $\mathscr{K}_{\infty}=\Sigma_{i} \mathcal{K}_{i}$.

Proof. Both $\mathcal{K}_{\infty}$ and $\sum_{i} \mathcal{K}_{i}$ contain $\Re$ and both have the same orthogonal complement under $B_{f}$. Hence they have the same dimension. Q.E.D. 
Corollary. Let $f_{\infty}=f \mid \mathcal{K}_{\infty}$. Then the $K_{\infty}$ orbit of $f_{\infty}$ is an affine subspace of $\mathscr{K}_{\infty}^{*}$

Now we once again assume that $G$ is nilpotent. Let $f_{\infty}=f \mid \mathcal{K}_{\infty}$ and let $U^{\infty}$ be the irreducible representation of $K_{\infty}$ corresponding to $f_{\infty}$ under the Kirillov correspondence.

THEOREM 2. ind $\left(K_{\infty}, G, U^{\infty}\right)$ is irreducible and corresponds to $\theta(f)$ under the Kirillov correspondence. Also $U^{\infty}$ is square integrable modulo its kernel.

Proof. From the above corollary $f_{\infty}$ has a flat orbit so it follows from the Moore-Wolf theorem [4] that $U^{\infty}$ is square integrable modulo its kernel.

To prove the irreducibility let $\mathcal{K}_{1}(f)$ and $\mathcal{K}_{1}(f)$ be as before. Let $H_{1}$ and $K_{1}$ be the corresponding subgroups. Let $K_{\infty, 1}=K_{\infty}\left(f \mid \mathcal{K}_{1}\right)$. $K_{1}$ fixes $f \mid \mathcal{H}_{1}$ so $K_{1}$ normalizes $\mathcal{H}_{\infty}$ and hence $K_{1}$ normalizes $K_{\infty, 1}$. From Corollary 3 it follows that $K_{\infty}=K_{1} K_{\infty, 1}$. Let $U^{\infty, 1}$ be the representation of $K_{\infty, 1}$ corresponding to $f \mid \Re_{\infty, 1}$ and let $U^{1}=\operatorname{ind}\left(K_{\infty, 1}, H_{1}, U^{\infty, 1}\right)$. By induction $U^{1}$ is irreducible and corresponds to $f_{1}=f \mid \mathcal{K}_{1}$. Let $L$ be the stabilizer of $U^{1}$ in $G$-i.e.

$$
L=\left\{x \in G \mid U^{1}\left(x \cdot x^{-1}\right) \approx U^{1}\right\}
$$

$x \in L$ if $\operatorname{ad}^{*} x\left(f_{1}\right)$ is in the $H_{1}$ orbit of $f_{1}$-i.e. iff $\operatorname{ad}^{*} x\left(f_{1}\right)=\operatorname{ad}^{*} y\left(f_{1}\right)$ for some $y \in H_{1}$. This is equivalent to saying $\operatorname{ad}^{*} y^{-1} x\left(f_{1}\right)=f_{1}$-i.e. $x \in K_{1}^{\prime} H_{1}$ where $K_{1}^{\prime}=\left\{x \in G \mid \operatorname{ad} x\left(f_{1}\right)=f_{1}\right\}$. It follows from standard arguments that $K_{1}^{\prime}$ is connected (see [1, I.3.3], e.g.) and that the Lie algebra of $K_{1}^{\prime}$ is precisely $\mathcal{K}_{1}^{f}=\mathscr{K}_{1}$. Hence $K_{1}^{\prime}=K_{1}$ and $L=K_{1} H_{1}$. $U^{1}$ extends to a representation $V$ of $L$. In fact, $K_{\infty}=K_{1} K_{\infty, 1} \subset L$. Let $V=\operatorname{ind}\left(K_{\infty}, L, U^{\infty}\right)$. Since $L=K_{1} H_{1}$, $V \mid H_{1}=\operatorname{ind}\left(K_{\infty, 1}, H_{1}, U^{\infty, 1}\right)=U^{1}$. It now follows from Mackey's theorem [3] that ind $(L, G, V)$ is irreducible. From the theorem on inducing in stage this is just ind $\left(K_{\infty}, G, U^{\infty}\right)$. It is obvious that this representation corresponds to $f$ -simply pick a polarization for $f$ contained in $\mathcal{K}_{\infty}$. Q.E.D.

\section{REFERENCES}

1. P. Bernat et al., Represéntations des groupes de Lie résolubles, Dunod, Paris, 1972.

2. L. Corwin, F. Greenleaf and R. Penney, A general character formula for the irreducible projections on $L^{2}$ of a nilmanifold (preprint).

3. G. W. Mackey, The theory of group representations, Lecture notes prepared by Dr. Fell and Dr. Lowdenslager, Math. Dept., Univ. of Chicago, Chicago, Ill., 1955. MR 19, 117.

4. C. Moore and J. Wolf, Square integrable representations of nilpotent groups, Trans. Amer. Math. Soc. 185 (1973), 445-462 (1974). MR 49 \# 3033.

Department of Mathematics, Purdue University, West Lafayette, Indiana 47907

Current address: Department of Mathematics, University of California, Berkeley, California 94720 